\title{
Free-electron laser induced processes in thin molecular ice
}

\author{
Bjorn Siemer, Sebastian Roling, Robert Frigge, Tim Hoger, Rolf Mitzner† \\ and Helmut Zacharias*
}

Intermolecular reactions in and on icy films on silicate and carbonaceous grains constitute a major route for the formation of new molecular constituents in interstellar molecular clouds. In more diff use regions and in protoplanetary discs, energetic radiation can trigger reaction routes far from thermal equilibrium. As an analog of interstellar ice- covered dust grains, highly-oriented pyrolytic graphite (HOPG) covered with $\mathrm{D}_{2} \mathrm{O}$, $\mathrm{NO}$, and $\mathrm{H}$ atoms is irradiated by ultrashort XUV pulses and the desorbing ionic and neutral products are analysed. The yields of several products show a nonlinear intensity

dependence and thus enable the elucidation of reaction dynamics by two-pulse correlated desorption.

\section{Introduction}

Molecular ice layers on grain particles are active environments for the trapping, formation, and release of new and even complex molecular species into the interstellar medium. ${ }^{1,2}$ In dense molecular clouds at low temperatures ( $\mathrm{T}_{\mathrm{s}} \mathrm{rv} 10$ to $50 \mathrm{~K}$ ), physisorbed atoms and molecules may undergo exothermic reactions to form new species. In particular the formation of water and of molecular hydrogen is believed to take place under such conditions..$^{3-8}$ Inner cores of large molecular clouds in the interstellar medium (ISM) are largely opaque to radiation in the visible, ultraviolet (UV), and vacuum ultraviolet (VUV) spectral range, and only partially transparent to IR radiation. ${ }^{9}$ Although only a low level of VUV and extreme ultraviolet (XUV) radiation is present ${ }^{10}$ due to the interaction of cosmic rays with the constituents of the clouds, its impact on the desorption of simple and complex molecules is relevant.

At the outer rims of such clouds and particularly in the photodissociation regions, the temperatures rise and the densities are considerably lower. ${ }^{1}$ In these regions more energetic photons can penetrate into the clouds and reach the surface of ice-covered grains with temperatures of up to about $100 \mathrm{~K}$. Such

Physikalisches Institut, Westfälische Wilhelms-Universität, 48149 Münster, Germany. E-mail: hzach@ uni-muenster.de

† present address: Helmholtz-Zentrum Berlin für Materialien und Energie, Berlin. 
conditions also prevail in the outer regions of approximately solar-mass protoplanetary discs at distances of about 3 to 50 AU from the central object. ${ }^{11,12}$ Typical grains consist of silicate and carbonaceous cores, and show sizes in the range from a few $\mathrm{nm}$ for polycyclic aromatic hydrocarbons (PAHs) to $1000 \mathrm{~nm}$ for amorphous silicates. ${ }^{13-15}$ With graphite and graphene-like surfaces the (photo) chemistry on some of these grains can be simulated in laboratory experiments.

Figure 1 shows schematically the typical processes that might occur on such grains when they interact with UV/VUV radiation. These basic processes have been described in various review articles. ${ }^{1,2,4,8,12}$ Besides the direct photodesorption of molecules adsorbed on the icy surface, more involved processes can be triggered. Photodissociation of 院 $1 m$ constituents generates energetic fragments which may directly leave or induce, via collisions, other molecules to leave the grain particle. Furthermore, reactions between dissociation products and constituents of the ice layer or even of the grain core may occur. The high kinetic energy of the primary dissociation products and the solid state density of the 院 $1 \mathrm{~m}$ enable reaction cascades very diff erent from equilibrium gas phase chemistry.

The interaction of VUV radiation with condensed molecular 院lms has been studied in recent years extensively by various groups. Early studies involved broad band VUV radiation from hydrogen and helium discharge lamps, ${ }^{16,17}$ while recently tunable radiation from frequency doubled lasers ${ }^{18}$ and synchrotron light sources ${ }^{19}$ has been employed. Besides simple monomolecular ices like $\mathrm{H}_{2} \mathrm{O}, \mathrm{CO}$ and $\mathrm{N}_{2},{ }^{20}$ ice 院lms of mixed constituents address issues of radical reactions in these 院lms. ${ }^{21}$ A prototypical radical is nitric oxide which has been observed in the gas phase of clouds ${ }^{22}$ and which, according to simulations, builds up to signi院cant concentrations in ices. ${ }^{23}$ It also is the main precursor for nitrogen and oxygen chemical networks. ${ }^{24,25}$ Such reactions become more important azer the incorporation of more complex molecules, like benzene, ${ }^{18}$ small polycyclic aromatic hydrocarbons, ${ }^{26}$ and $\mathrm{C}_{60}$ fullerenes ${ }^{27}$ as dopants into water ice.

Important aspects of VUV and XUV induced processes in condensed molecular 院 $1 \mathrm{~ms}$ are further addressed by low-energy electron beam irradiation of these systems. Such electrons are also liberated by XUV irradiation of the samples. The

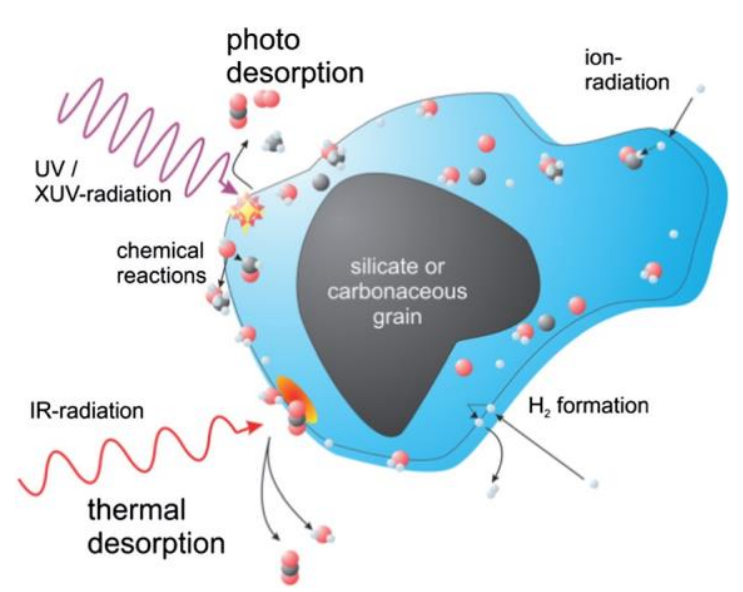

Fig. 1 Illustration of reactive processes on icy grains. 
primary ions, either generated directly by the photons or by secondary photoelectrons, may initiate very eff ective ion-molecule reactions such as protonation

of photoproducts and original constituents. Electron stimulated desorption of various hydrogen and oxygen containing positive ions ${ }^{28}$ and aromatic molecules ${ }^{29}$ from water ice has been reported, as well as the interaction with more complex ices. $^{30}$

In this paper we report on preliminary studies of the interaction of XUV photons with graphite surfaces covered by hydrogen atoms, nitric oxide, or water ice. We make use of the ultrashort duration of XUV pulses from a free-electron laser, of the order of $30 \mathrm{fs}$, to shed light onto reaction pathways triggered by this radiation. Although the photon energy of these pulses is certainly higher than that from external irradiation sources of molecular clouds due to the atomic hydrogen ionization limit, the basic dynamic features induced by this radiation in the ice 院lms are expected to be similar to the action of VUV photons. Moreover, internal radiation created by cosmic ray collisions produce XUV radiation, even in dense clouds.

\section{Experimental}

The experiments were performed at beamlines 1 and 3 of the free-electron laser at Hamburg (FLASH), which provides pulsed radiation in the XUV spectral range with fundamental photon energies from about hn $1 / 425$ to $300 \mathrm{eV} .{ }^{31} \mathrm{In}$ the present experiments, FLASH operated at photon energies of hn 1/4 $38.15 \mathrm{eV}\left(1 \frac{1}{4} 32.5 \mathrm{~nm}\right)$ and $57.1 \mathrm{eV}\left(1 \frac{1}{4} 21.7 \mathrm{~nm}\right)$ in a $5 \mathrm{~Hz}$ single bunch mode. The spectral energy width averaged to about $\mathrm{hDn} \mathrm{rv} 0.8 \mathrm{eV}$, with pulse energies up to about $(13.5 \pm 3.5) \mathrm{mJ}$. The average pulse duration was measured to ca. $t_{L} r v 30 \mathrm{fs}^{32}$

For time-resolved experiments, a wave front dividing beam splitter and pulse split delay unit (SDU) was employed. ${ }^{33,34}$ This SDU provides two jitter-free replica pulses for XUV pump-XUV probe experiments (see Fig. 2a). The incoming FLASH beam is split into two parts by the 院rst mirror which is partially moved into the beam path. Each partial beam propagates on a separated beam path (orange and green) and both are recombined again into the original direction with the 院nal mirrors of their respective beam paths. Following recombination, the soray beams are focused by a toroidal mirror to ellipsoidal spot sizes of $220 \mathrm{~mm} \times 175$ $\mathrm{mm}$ and $235 \mathrm{~mm} \times 125 \mathrm{~mm}$ for the variable and 院xed beam paths, respectively. The spatial overlap of the two foci is monitored by an x-ray CCD camera in the equivalent focal position via a re寗ecting quartz plate which is brought into the beam path. In this way the focal diameter has been measured and the stability of the spatial overlap ensured over the whole delay range. This set-up further facilitated a measurement of the diff erent intensities in the two focal spots. The

p- polarized XUV pulses strike the highly oriented pyrolytic graphite (HOPG) surface at an angle of incidence of $\mathrm{q} 1 / 467.5^{\circ}$ relative to the surface normal. At these photon energies of $38.15 \mathrm{eV}$ and $57.1 \mathrm{eV}$ and incident angle, about $42 \%$ and $32 \%$ of the incident radiation is re蜜ected from graphite and about $12 \%$ and $5 \%$ from thick ice 院lms, respectively. ${ }^{35,36}$

Figure $2 \mathrm{~b}$ shows schematically the set-up for the detection of desorbing species. The HOPG sample exhibited a mosaic angle of $0.4^{\circ}$ and a diameter of $10 \mathrm{~mm}$. It was mounted on a liquid nitrogen containing reservoir attached to a holder on an $\mathrm{X}-\mathrm{Y}-\mathrm{Z}$ stage in an ultra-high vacuum (UHV) chamber. The UHV 


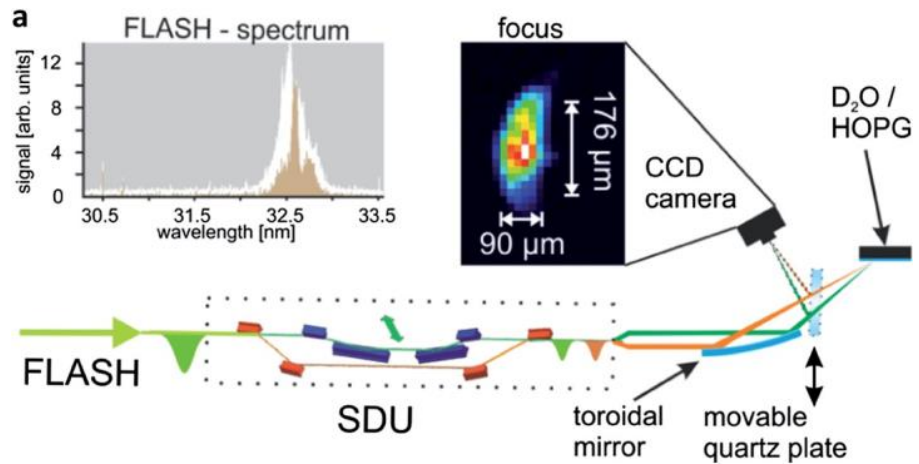

b

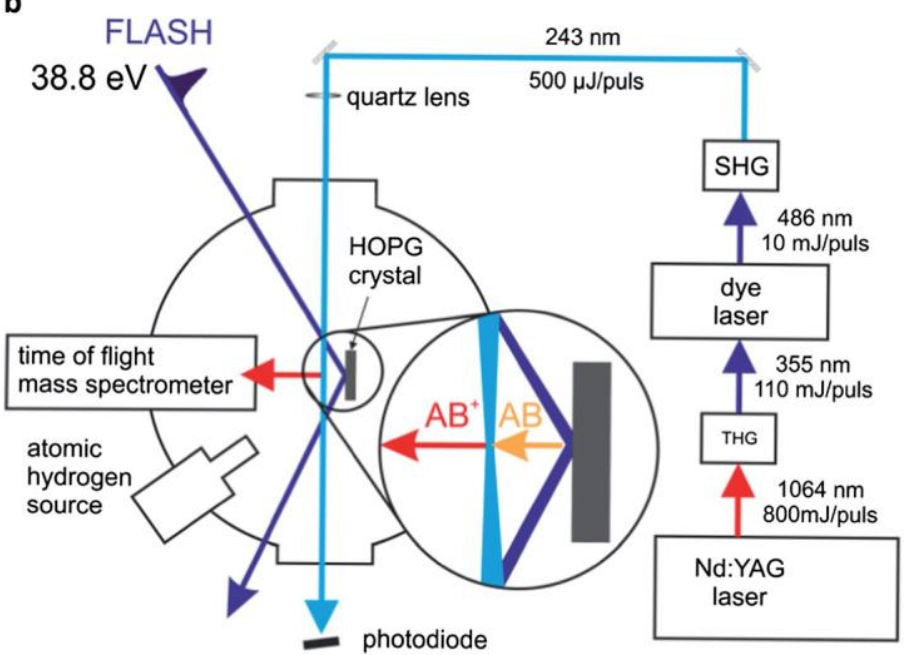

Fig. 2 (a) Schematic diagram of the optical layout of the experimental set-up. The spectrum shows in dark colour a single shot spectrum and in white the spectral envelop over 1000 pulses. (b) Experimental set-up for the detection of desorption products.

chamber was evacuated by an oil-free pumping system to a base pressure of $<7 \$$ $10^{-10}$ mbar. The HOPG sample was prepared by cleaving with adhesive tape and long-time annealing at $800 \mathrm{~K}$ by electron bombardment from the rear side. A sample preparation the temperature of the graphite crystal was held at $T_{\mathrm{s}} 1 / 495 \mathrm{~K}$, as monitored with a K-type thermocouple attached to the surface.

Molecules were dosed onto the HOPG surface through a tube at normal incidence at a constant chamber pressure for several minutes or via atomic and molecular beams. Hydrogen and deuterium atoms were supplied by a heated tungsten capillary, using a design which avoids ionic species. Hydrogen only sticks as chemisorbed atomic hydrogen azer surmounting a considerable adsorption barrier of $E_{b} r v 200 \mathrm{meV}^{37,38}$ an energy which can be achieved when heating the tube to temperatures above $2000{ }^{\circ} \mathrm{C}$. NO molecules were dosed by a doubly diff erentially pumped pulsed thermal molecular beam. Nitric oxide

condenses as dimers in a self-terminating monolayer. At this surface temperature water condenses as microporous amorphous ice. For water ice, coverages of 80 to $200 \mathrm{ML}$ have been prepared and investigated. At XUV photon energies of $38 \mathrm{eV}$ 
and $57 \mathrm{eV}$ the penetration depth of the radiation into ice amounts to about $9 \mathrm{~nm}$ and $15.7 \mathrm{~nm}$, respectively. ${ }^{35,36}$ Therefore, at an incident angle of $q^{1 / 4} 67.5^{\circ}$ the 院rst 11 and $18 \mathrm{ML}$, respectively, are irradiated using a value of $1.15 \$ 10^{15}$ molecule $\mathrm{cm}^{-2}$ for a monolayer (ML) of water on graphite.

Cations desorbing directly arer irradiation by the XUV pulse were measured by a Wiley-McLaren type time-of-蜜ight mass spectrometer (ToF) mounted along the surface normal direction. Then the sample holder served as the 院rst repelling electrode. Desorbing neutral molecules were 院rst ionized by tuneable laser radiation (see Fig. 2b) and then detected by the same ToF spectrometer. The output of the microchannel plates was directly monitored on a digital oscilloscope, gated and forwarded to a computer. For neutral hydrogen atoms a $(2+1)$ REMPI scheme via the two-photon excitation $2 \mathrm{~s}^{2} \mathrm{~S}_{1 / 2}$ ) $1 \mathrm{~s}{ }^{2} \mathrm{~S}_{1 / 2}$ at $1 \mathrm{rv} 243 \mathrm{~nm}$ was chosen, and for NO a $(1+1)$ REMPI scheme via the $\left.\mathrm{A}^{2} \mathrm{~S}^{+}\right) \mathrm{X}^{2} \mathrm{P}$ g-band transitions around $1 \mathrm{rV} 226 \mathrm{~nm}$. This UV probe laser radiation was provided by a frequency doubled, tuneable dye laser with nanosecond pulse duration, which was electronically synchronized to the FEL pulses.

For each delay time setting between the desorbing XUV and the detecting tuneable dye laser pulses, the time-of-䀄ight mass spectra were summed over 1000 single shots. For each shot the signal and the relative FLASH pulse energy as given by the gas monitor detector was stored. Normalization and averaging were performed in the data analysis.

\section{Results}

\section{Water ice}

XUV photon induced reactions in undoped $\mathrm{D}_{2} \mathrm{O}$ water ice multilayers with thicknesses of up to about $200 \mathrm{ML}$ are observed. At the photon energies employed, $38.15 \mathrm{eV}$ and $57.1 \mathrm{eV}$, the exciting radiation is absorbed in the top most ice layers and does not reach the substrate. At respective penetration depths of 9.0 and $15.7 \mathrm{~nm}$ into the ice and an angle of incidence of $\mathrm{q} 1 / 467.5^{\circ}$, only the top most 11 and $18 \mathrm{ML}$ of the ice, respectively, are irradiated. A illuminating the surface with so x-ray radiation at hn $1 / 438.15 \mathrm{eV}\left(1 \frac{1}{4} 32.5 \mathrm{~nm}\right)$ with pulse energies up to (13.5 \pm 3.5$) \mathrm{mJ}$, the directly desorbing cations were detected with a time-of-蜜ight mass spectrometer. When reducing the acceleration voltage in the spectrometer, an asymmetric line shape for the detected ions arises and thus the kinetic energy distribution of these ions can be derived.

A typical mass spectrum is shown in Fig. 3. Besides the expected masses of $\mathrm{D}_{2} \mathrm{O}^{+}, \mathrm{OD}^{+}, \mathrm{O}^{+}$, and $\mathrm{D}^{+}$resulting from fragmentation and ionization, products of reactions within the ice layers are also observed, in particular $\mathrm{D}_{3} \mathrm{O}^{+}, \mathrm{D}_{2} \mathrm{O}_{2}^{+}$, and $\mathrm{O}_{2}^{+}$. In addition, water cluster ions $\left(\mathrm{D}_{2} \mathrm{O}\right)^{+}$and deuteronated clusters $\left(\mathrm{D}_{2} \mathrm{O}_{2}\right) \mathrm{D}^{+}$ which are formed by dissociative deuteronation are observed at higher masses. ${ }^{39}$ The yield of these reactively formed products exhibits a nonlinear dependence on the FEL intensity which is exemplarily shown in Fig. 4 for $\mathrm{O}_{2}{ }^{+}$ions. The signals can be 院tted by a power law, Y rv I ${ }^{\mathrm{n}}$, where $\mathrm{n} 1 / 43$, even for unfocussed FEL pulses. In an oversimpli院ed picture, such a power dependence then suggests that three XUV photons are involved in the formation of the product species. Strong pulseto-pulse 蜜uctuations of the FEL output power causes a broad scatter of single shot data, which leaves some variations in the exponent $n$. 


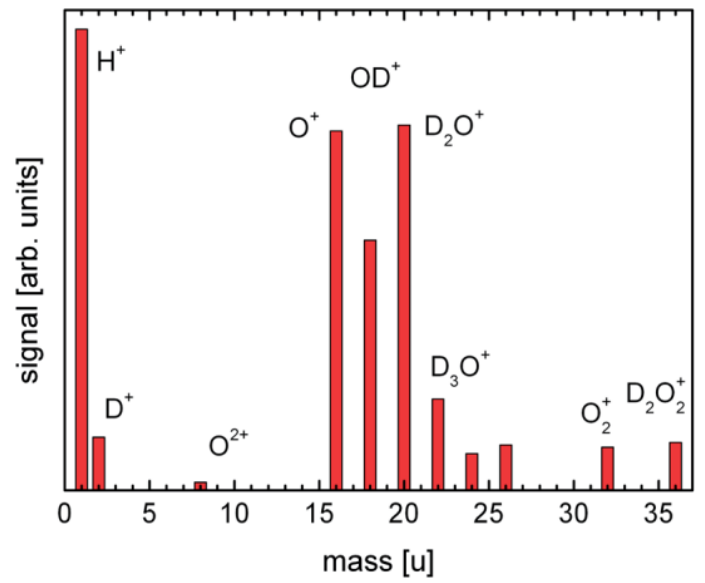

Fig. 3 Time-of-flight mass spectrum of species desorbed form ice/graphite by XUV FEL pulses at hn $1 / 438.15 \mathrm{eV}$.

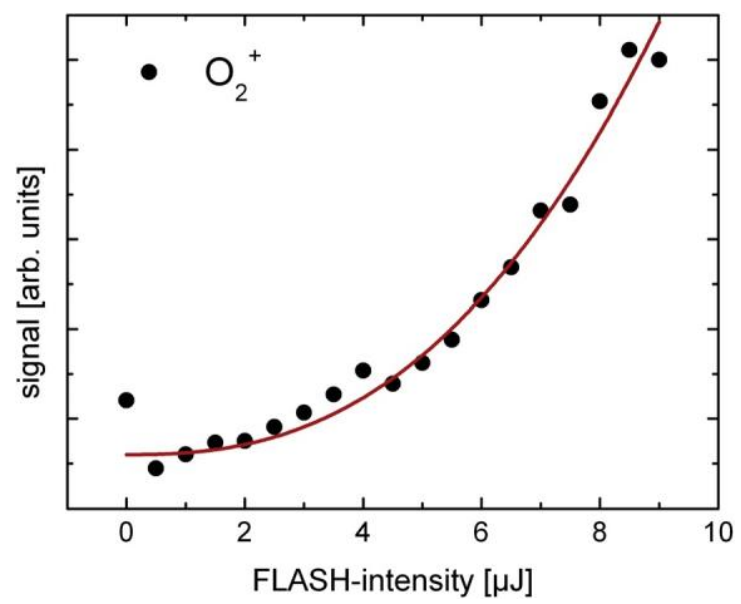

Fig. 4 Dependence of the $\mathrm{O}_{2}{ }^{+}$ion signal on the pulse energy of the desorbing FEL pulses at $h n 1 / 457.1 \mathrm{eV}$. The other oxygen containing ions observed (Fig. 5) show similar intensity dependencies.

Such a nonlinear intensity dependence allows the investigation of the temporal development of the signal via an XUV pump-XUV probe set-up, thus partially elucidating the reaction dynamics. For the present experiment the SDU was employed with an asymmetric intensity splitting of the FEL pulse. In the overlapping foci of both beams this resulted in an averaged intensity of $(6.5 \pm 1.2) \$ 10^{10} \mathrm{~W} / \mathrm{cm}^{2}$ and $(1.1 \pm 0.1) \$ 10^{11} \mathrm{~W} / \mathrm{cm}^{2}$ for the variable and 院xed beam, respectively. Thus, an asymmetric yield is expected as a function of temporal delay between both pulses. For each delay, Dt, the spatial overlap of both beams was controlled by measuring the beam pro院le by re蜜ecting the beam off a quartz plate onto a CCD camera positioned at the equivalent focal position before entering the experimental chamber (see Fig. 2a). 
Figure 5 shows the yield of various product ions as a function of delay times Dt $1 / 4-1$ and +5 ps between both XUV pulses. The data points are averages of several runs. Besides the expected $\mathrm{D}_{2} \mathrm{O}^{+}$ions, several fragment ions $-\mathrm{D}^{+}, \mathrm{O}^{+}$, and $\mathrm{OD}^{+}$- and reaction products $-\mathrm{D}_{2} \mathrm{O}_{2}{ }^{+}, \mathrm{O}_{2}{ }^{+}$, and $\mathrm{D}_{3} \mathrm{O}^{+}$(see Fig. 6) - are also observed. The temporal structure of the two-pulse correlation curves are very similar for all ions shown. Fitting the longer main signal by a Gaussian curve yields a maximum at Dt $1 / 42.2 \mathrm{ps}$ and a signal width of about $2.7 \mathrm{ps}$ (FWHM). In addition, for the oxygen containing products, a second maximum is observed for Dt $1 / 40.6$ ps. Despite the fact that this relative maximum is supported by just one data point, it was observed in every run. The very limited beam time at the FEL prohibited a 院ner temporal grid. It is worth noting that the signal of nearly all ions, including the $\mathrm{D}^{+}$ion, approach zero for longer temporal delays. The $\mathrm{D}_{2} \mathrm{O}^{+}$ion signal, however, shows a large background signal of more than $50 \%$ of the peak signal which does not change with delay. A large part of this signal is thus generated by a single photon process, as expected.

From the observation that the maximum of the signals do not occur when both pulses overlap in time, it may be concluded that not only pure photonic processes are involved in forming these cations. Instead, collisions between fragments

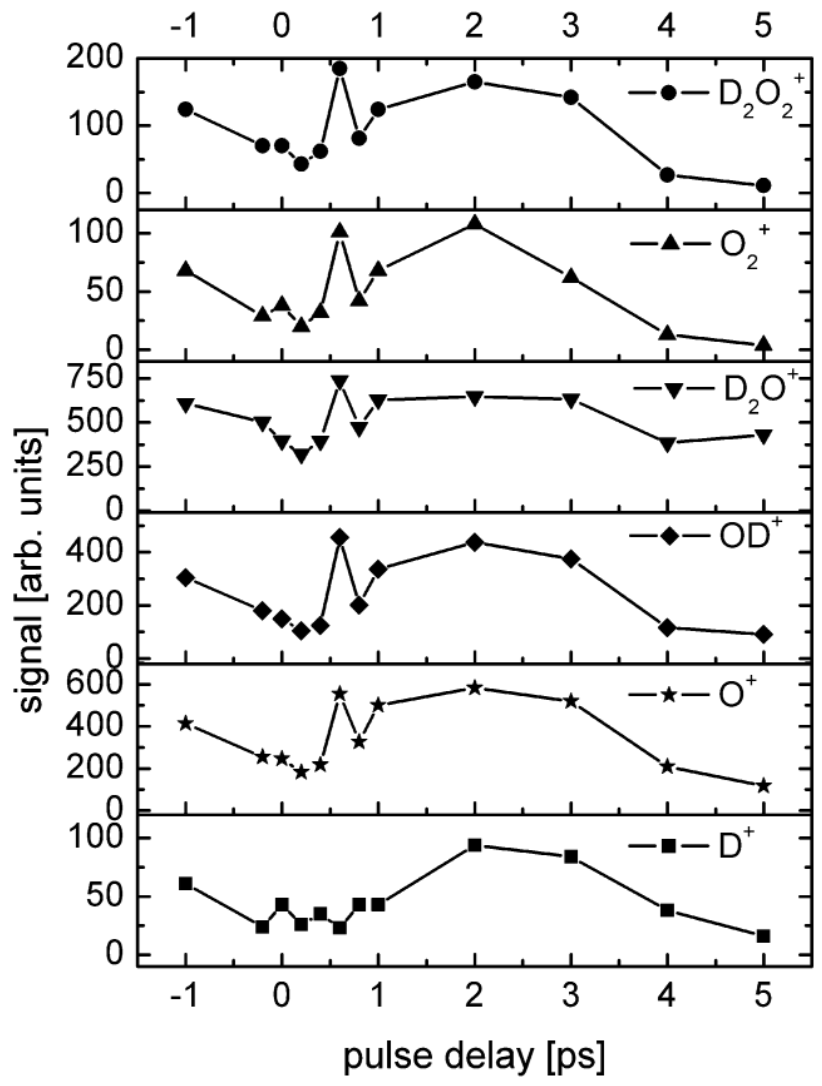

Fig. 5 Two-pulse correlated XUV desorption of various cation fragments from $\mathrm{D}_{2} \mathrm{O}$ ice on graphite. The photon energy of the desorbing laser was $\mathrm{hn} 1 / 438.15 \mathrm{eV}$. 


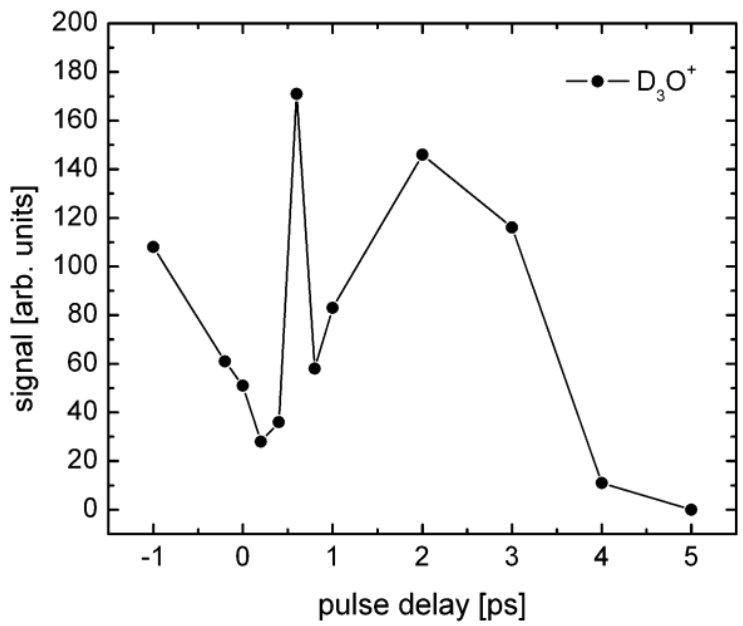

Fig. 6 Two-pulse correlated XUV desorption of $\mathrm{D}_{3} \mathrm{O}^{+}$from ice/graphite. The photon energy of the desorbing laser was $\mathrm{hn} 1 / 438.15 \mathrm{eV}$.

within the ice layer appear to be important. The 院rst step for the generation of these products certainly involves the photodissociation of a water molecule:

$$
\begin{aligned}
& \mathrm{D}_{2} \mathrm{O}+\mathrm{hn}(38.15 \mathrm{eV}) / \mathrm{D}_{2} \mathrm{O}^{+}+\mathrm{e}^{-}\left(\mathrm{E}_{\mathrm{kin}}{ }^{1 / 4} 25.55 \mathrm{eV}\right)^{40} \\
& \zeta O D+D\left(E_{\text {exc }} 1 / 433.05 e V\right)^{43} \\
& \angle O D+D^{+}+e^{-}\left(E_{e x c} 1 / 419.45 e V\right)^{41} \\
& \left\langle\mathrm{OD}^{+}+\mathrm{D}+\mathrm{e}^{-}\left(\mathrm{E}_{\mathrm{exc}}{ }^{1 / 4} 19.96 \mathrm{eV}\right),{ }^{41}\right.
\end{aligned}
$$

where $E_{\text {kin }}$ and $E_{\text {exc }}$ denote the kinetic energy of the liberated electron and the excess energy available for distribution over internal and translational degrees of freedom, respectively.

Since all products detected in Figs. 5 and 6, except $\mathrm{D}_{2} \mathrm{O}^{+}$, show a yield close to zero at large pulse separations in the two-pulse correlation, these products are not generated in a single photon event. The hydroxyl radicals produced in the beam path of the FEL beam penetrating the ice layer may then collide with each other and form hydrogen peroxide:

$$
\mathrm{OD}+\mathrm{OD}+\mathrm{M} / \mathrm{D}_{2} \mathrm{O}_{2}+\mathrm{M}
$$

where also substrate phonons can act as the third collision partner to carry away the excess energy of the reaction.

The temporal behaviour of the two-pulse correlation signals suggests that hydrogen peroxide serves as a reservoir molecule for the other products. A second photon then either ionizes hydrogen peroxide, produces the other fragments, or strips off both hydrogen atoms:

$$
\mathrm{D}_{2} \mathrm{O}_{2}+\mathrm{hn} / \mathrm{D}_{2} \mathrm{O}_{2}^{+}+\mathrm{e}^{-}\left(\mathrm{E}_{\mathrm{kin}}{ }^{1 / 4} 27.5 \mathrm{eV}\right)^{42}
$$




$$
\begin{aligned}
& \gamma \mathrm{OD}+\mathrm{OD}\left(\mathrm{E}_{\mathrm{exc}} 1 / 435.92 \mathrm{eV}\right)^{43} \\
& O D^{+}+O D+e^{-}\left(E_{e x c} 1 / 422.8 e V\right)^{42} \\
& \text { / } \mathrm{DO}_{2}+\mathrm{D}\left(\mathrm{E}_{\mathrm{exc}} 1 / 434.32 \mathrm{eV}\right)^{43} \\
& / \mathrm{DO}_{2}{ }^{+}+\mathrm{D}+\mathrm{e}^{-}\left(\mathrm{E}_{\mathrm{exc}}{ }^{1 / 4} 22.79 \mathrm{eV}\right)^{42} \\
& \mathrm{O}_{2}+\mathrm{D}+\mathrm{D}\left(\mathrm{E}_{\mathrm{exc}} 1 / 432.22 \mathrm{eV}\right)^{44} \\
& / \mathrm{O}_{2}+\mathrm{D}^{+}+\mathrm{D}+\mathrm{e}^{-}\left(\mathrm{E}_{\mathrm{exc}} 1 / 419.62 \mathrm{eV}\right)^{42} \\
& / \mathrm{O}_{2}{ }^{+}+\mathrm{D}+\mathrm{D}+\mathrm{e}^{-}\left(\mathrm{E}_{\text {exc }}{ }^{1 / 4} 20.15 \mathrm{eV}\right)^{45,46}
\end{aligned}
$$

In the gas phase the three product channels show similar reaction probabilities in the VUV. ${ }^{43}$ This second step may be followed by the absorption of a third photon which then yields the corresponding atomic and molecular ions, if they are not already produced in the previous step.

$$
\begin{aligned}
& \mathrm{OD}+\mathrm{hn} / \mathrm{OD}^{+}+\mathrm{e}^{-}\left(\mathrm{E}_{\mathrm{kin}} 1 / 425.13 \mathrm{eV}\right)^{47} \\
& D+h n / D^{+}+e^{-}\left(E_{k i n} 1 / 424.55 e V\right)^{48} \\
& \mathrm{O}_{2}+\mathrm{hn} / \mathrm{O}_{2}{ }^{+}+\mathrm{e}^{-}\left(\mathrm{E}_{\text {kin }}{ }^{1 / 4} 26.08 \mathrm{eV}\right)^{45}
\end{aligned}
$$

The nonlinear yield dependence, which is equal or larger than $n 1 / 43$, suggests that this last step indeed occurs.

In this reaction system, process (2) which describes the formation of hydrogen peroxide, is certainly the step which governs the temporal evolution of the signal. Since the excess energy release in processes (1a) through $\left(1 \mathrm{a}^{00}\right)$ is high, with a maximum kinetic energy in the OD radicals of up to $E_{\text {kin }} 1 / 43.3 \mathrm{eV}$, neighbouring hydroxyl radicals can even react within the temporal overlap of both FEL pulses at Dt $1 / 40$ ps. This part then contributes to the small signal at time zero. However, it is evident that it requires some more time to accumulate an optimal concentration of hydrogen peroxide. The two-pulse correlation results (Fig. 5) suggest that this occurs at about Dt $1 / 42.2$ ps for hydroxyl radicals, presumably produced in the bulk ice. Surface hydroxyl may react on a faster time scale as is suggested by the peak observed at Dt 1/4 0.6 ps. This mechanism requires at least two XUV photons from the FEL beam, in accordance with the recorded power dependencies of $n \$ 3$. Hydrogen peroxide is thus an essential intermediate precursor for the formation of oxygen containing ions. This conclusion is supported by earlier experiments where ice layers have been irradiated by electron beams with kinetic energies in the same region as the photon energies employed here. ${ }^{49,50}$

Other oxygen containing cations observed, as shown in Fig. 5, may have additional channels which contribute to the signal height. In particular, $\mathrm{D}_{2} \mathrm{O}^{+}$ shows a large background of about one half of the peak intensity, when both pulses are separated in time. This can easily be understood by the direct and dissociative ionization of the ice constituents. It is interesting to note that for $\mathrm{D}^{+}$, the fast peak at Dt $1 / 40.6$ ps is missing. A direct $\mathrm{D}^{+}$ion abstraction seems not to take place. Moreover, since we observe a signi院cant two-pulse correlation signal, 
which can only be observed for a nonlinear intensity dependence, a multiphoton process must also be invoked for this product channel.

The hydronium cation, $\mathrm{D}_{3} \mathrm{O}^{+}$, which is also observed to show a pronounced temporal dependence in two-pulse correlated desorption (see Fig. 6), is easily formed by deuteronation reactions. For this product, a fast correlation peak arer ca. Dt $1 / 40.6 \mathrm{ps}$ is also observed, followed by a major peak at about Dt rv $2.2 \mathrm{ps}$. This is again interpreted as a distinction between surface and bulk reactions. The relative integrated yield of the surface contributions is about 0.09 .

In this case either a process via the $\mathrm{D}^{+}$ion

$$
D^{+}+D_{2} \mathrm{O}+M / D_{3} \mathrm{O}^{+}+M
$$

or a reaction like

$$
\mathrm{D}_{2} \mathrm{O}^{+}+\mathrm{D}_{2} \mathrm{O} / \mathrm{D}_{3} \mathrm{O}^{+}+\mathrm{OD}
$$

is conceivable. The $\mathrm{D}^{+}$ions observed (Fig. 5) display only the broad peak at Dt $1 / 4$ 2.2 ps. The $\mathrm{D}_{3} \mathrm{O}^{+}$ion products (Fig. 6) exhibit both peaks in the signal at $\mathrm{Dt} 1 / 40.6$ ps and at $2.2 \mathrm{ps}$. At longer pulse delays the $\mathrm{D}_{3} \mathrm{O}^{+}$signal approaches zero. This last observation argues against reaction (5b), because the generation of the water cation requires only a single XUV photon. This is an effi cient process also for widely separated pulses, as is witnessed by the large background in the temporal dependence of the $\mathrm{D}_{2} \mathrm{O}^{+}$signal (Fig. 5). The non-appearance of the fast peak at $\mathrm{Dt}$ $1 / 40.6$ ps in the $\mathrm{D}^{+}$ion signal (Fig. 5) remains to be explained. It is conceivable that these early $\mathrm{D}^{+}$ions react with other products or neutral 院 $1 m$ constituents, see reaction (5a), before they were able to leave the surface. Certainly, additional experiments are required and in particular the kinetic energy release into the diff erent channels need to be measured before a de院nite conclusion can

be drawn.

It is important to note that photoinduced processes within the bulk ice lead not only to the formation of new species, in this case hydrogen peroxide, but to the release of these species and dissociation products from the grain surface into the gas phase. Collisions occurring between photodissociation products and ice constituents are central to the formation of new species. Furthermore, even without reactions, such energetic collisions will lead to the desorption of neutral species from the ice layer, as has been observed for vibrational resonant IRinduced desorption from molecular ices, like $\mathrm{N}_{2} \mathrm{O},{ }^{51,52} \mathrm{CD}_{3} \mathrm{~F},{ }^{53}$ and methane. ${ }^{54}$

\section{Kinetic energies of directly desorbing ions}

As is evident from Fig. 5, the XUV pulses also induce the desorption of hydrogen ions from ice layers. In this case, the temporal behaviour of the two-pulse correlated desorption signal suggests that $\mathrm{D}^{+}$ions liberated in the bulk predominantly contribute to the signal observed. These hydrogen ions show a high kinetic energy as shown in Fig. 7, where both the velocity and kinetic energy distributions of $\mathrm{H}^{+}$are plotted. The velocity distribution in Fig. 7a can be 院tted by two Maxwellian distributions with respective temperatures of T $1 / 47000 \mathrm{~K}$ and $19000 \mathrm{~K}$ and relative integrated yields of $45 \%$ and $55 \%$. From this analysis, an average kinetic energy of $\mathrm{E}_{\mathrm{kin}} 1 / 41.8 \mathrm{eV}$ for the $\mathrm{H}^{+}$ions is obtained. When the 
a)

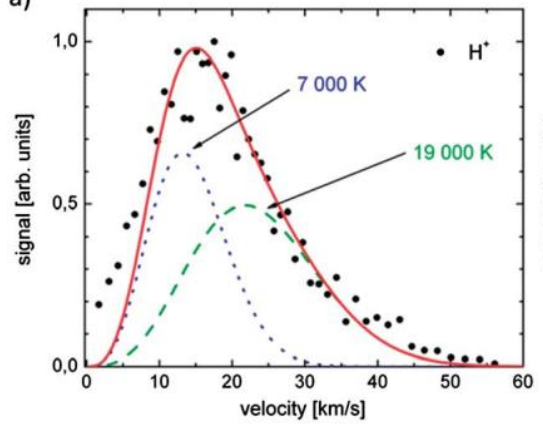

b)

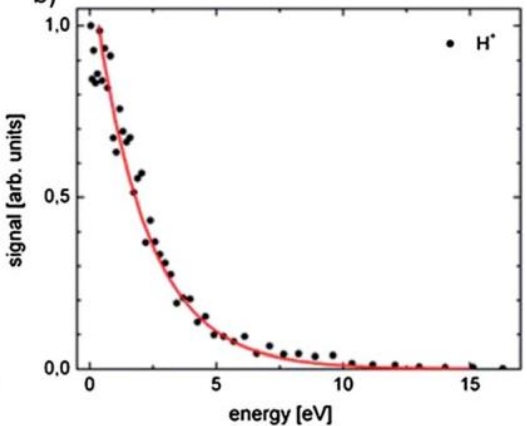

Fig. 7 (a) Velocity distribution of $\mathrm{H}^{+}$after XUV desorption from ice/graphite. Two Maxwellian velocity distribution with $\mathrm{T} 1 / 47000 \mathrm{~K}$ and $19000 \mathrm{~K}$ approximate most of the distribution. Some deviation at low velocities can be noticed. (b) Kinetic energy distribution of $\mathrm{H}^{+}$from ice/graphite. The red curve is a fit to a single sided exponential yielding a kinetic temperature of $16245 \mathrm{~K}$. Photon energy: $38.15 \mathrm{eV}$.

measured distribution is transformed into the energy picture (Fig. 7b) a singlesided exponential distribution, $\mathrm{W}\left(\mathrm{E}_{\mathrm{kin}}\right)$, is obtained

$$
W ð E_{k i n} \text { P r v a } 1^{\text {/pffi ffi ffffi }} \text { expf- } E_{k i n}=k T g \text { : }
$$

Such distributions are oren found in plasma physics to determine kinetic temperatures. In this case a best 院 t yields a kinetic temperature of $\mathrm{T}_{\text {kin }} 1 / 416245 \mathrm{~K}$, corresponding to an energy of about $\mathrm{E}_{\mathrm{kin}}{ }^{1 / 4} 2.1 \mathrm{eV}$. For $\mathrm{O}^{+}$and $\mathrm{O}_{2}$ ions, high kinetic energies of $0.84 \mathrm{eV}$ and $0.39 \mathrm{eV}$, respectively, have also been observed. ${ }^{55}$

\section{Neutral hydrogen}

Diff erent from other groups which investigate the reaction dynamics of phys- isorbed hydrogen on ice, ${ }^{4}$ graphite, ${ }^{56}$ and silicate surfaces ${ }^{7,57}$ we concentrate here on chemisorbed hydrogen atoms on a graphite substrate. Such conditions are reached in warmer parts of the ISM where molecular gases have already been desorbed from the grain particles. Such grain temperatures prevail, e.g., at the outer rims of molecular clouds and of protoplanetary discs. ${ }^{12}$

The high photon energy of the FEL radiation enables the direct desorption of ionized and neutral hydrogen species. For this experiment on HOPG (0001) we kept, arer cleaning, the surface temperature at room temperature to prohibit the adsorption of water from the UHV background gas. Hydrogen atoms were dosed onto the surface from a heated tungsten capillary. In this way the adsorption barrier of ca. $200 \mathrm{meV}$ can be overcome. In this experiment, a hydrogen coverage of about $0.25 \mathrm{ML}$ is adsorbed on the surface.

Neutral $\mathrm{H}$ atoms were desorbed by $\mathrm{hn} 1 / 438.8 \mathrm{eV}$ radiation using p-polarized FEL pulses with an average pulse energy of $13 \mathrm{~mJ}$, incident under an angle of $q 1 / 4$ $67.5^{\circ}$ relative to the surface normal. Slight focusing resulted in an illuminated area of about $0.19 \mathrm{~mm}^{2}$, an applied 蜜uence of $6.9 \mathrm{~mJ} / \mathrm{cm}^{2}$ and a 蜜uence absorbed by the substrate of about $3.9 \mathrm{~mJ} / \mathrm{cm}^{2}$. By changing the temporal delay between the probe and desorption laser pulses, an arrival time spectrum of the $\mathrm{H}$ atoms in the probe volume is recorded ${ }^{58}$ A er transformation into the velocity space, rather 


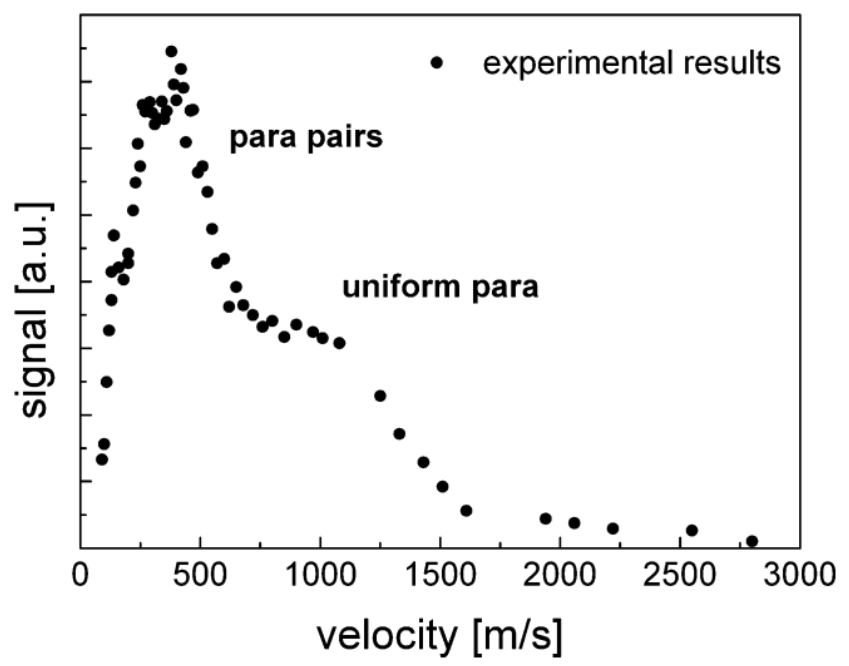

Fig. 8 Velocity distribution of neutral $\mathrm{H}$ atoms desorbing from chemisorbed $\mathrm{H}$ on graphite in a uniform para adsorbate configuration. This results in a coverage of $0.25 \mathrm{ML}$ H on graphite. Average kinetic energies of $\left\langle\mathrm{E}_{\mathrm{kin}}>1 / 40.8 \mathrm{meV}\right.$ and $5.3 \mathrm{meV}$ are deduced for the para pairs and uniform para configurations, respectively.

slow velocities for the desorbing hydrogen atoms are observed (see Fig. 8). A theoretical analysis of the desorption process reveals that multiple electron scattering on the $\mathrm{H}$ adsorbate causes the desorption, thus a typical DIMET (desorption induced by multiple electronic transitions) process is observed. ${ }^{59}$ These hot electrons are generated by the absorption of the high energy XUV photon by the graphite substrate, producing a dense cloud of hot electrons in the vicinity of the surface with a rather short lifetime of about half a picosecond. Multiple electron scattering leads to a successive population of higher vibrational states of the $\mathrm{C}-\mathrm{H}$ vibration, and, in a 院nal step, the desorption out of one of these vibrational states. A peak at a velocity of $\mathrm{v}^{1 / 4} 425 \mathrm{~ms}^{-1}$, and two shoulders at $255 \mathrm{~ms}^{-1}$ and $136 \mathrm{~ms}^{-1}$ are apparent. These very slow $\mathrm{H}$ atoms show an average kinetic energy of only $E_{\text {kin }} 1 / 40.8 \mathrm{meV}$. A second maximum at a velocity of $\mathrm{v} 1 / 41000$ $\mathrm{ms}^{-1}$ yields an average kinetic energy of $E_{\text {kin }} 1 / 45.3 \mathrm{meV}$. The relative integrated intensities of the very slow and the faster peak are about the same.

The theoretical analysis shows good agreement with the experimental observations when the very slow peak is caused by desorption of $\mathrm{H}$ atoms out of a hydrogen surface dimer pair which occupies the para position on the underlying graphene lattice..$^{38,60,61}$ The faster peak can be reconciled when one assumes that the desorption occurs out of a uniform para con院guration of adsorbed $\mathrm{H}$ atoms. ${ }^{58}$ In this con院guration all $\mathrm{H}$ atoms are only adsorbed in a para position relative to each other on the graphene lattice. Thereby additional energy is gained by the adsorbate system, as depicted in Fig. 9. There, the adsorption energies gained by both atoms when adsorbing a second hydrogen atom in the ortho-, para-, and uniform para-position relative to the 院rst one is shown. Besides a barrier-free adsorption, as for a para pair, the energy gain is even higher by about DE rv $0.6 \mathrm{eV}$ in the uniform para con院guration. Thus, instead of both hydrogen atoms exhibiting a binding energy of about $E_{b} r v 0.8 \mathrm{eV}$ each as for the adsorption of 


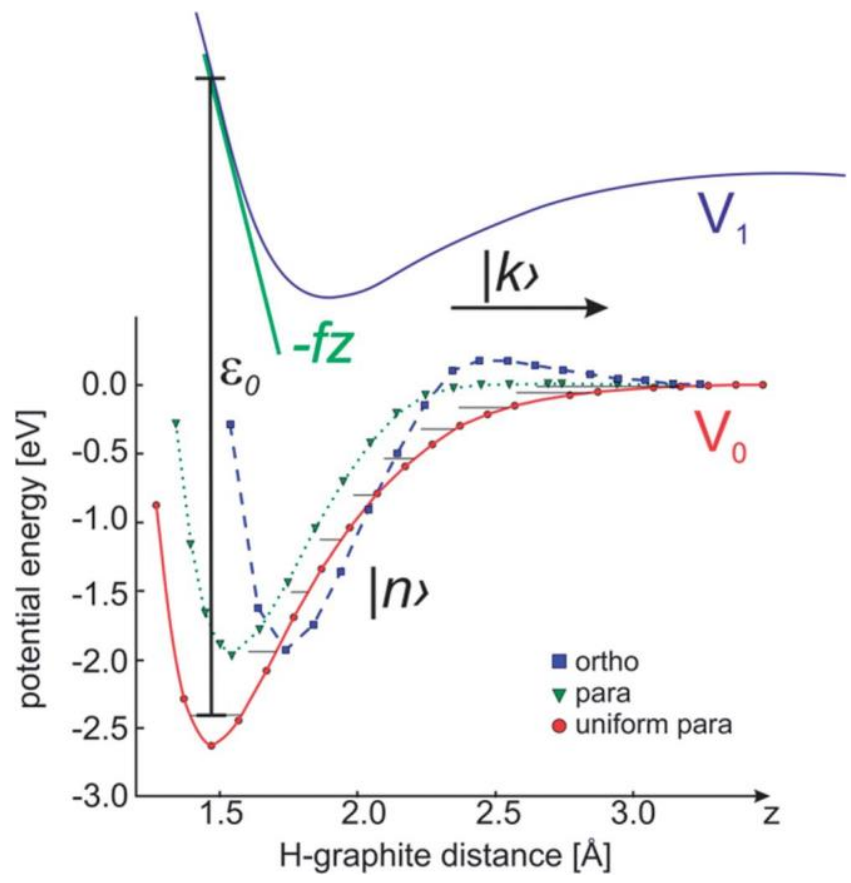

Fig. 9 Potential energy diagram for $\mathrm{H}$ atoms chemisorbed on graphene for the ortho-, para-, and uniform para connigurations.

single isolated $\mathrm{H}$ atoms, ${ }^{61}$ the uniform para pair show together an adsorption energy of $E_{b} r v 2.6 \mathrm{eV}$, thus $1.0 \mathrm{eV}$ larger than the sum of two single $\mathrm{H}$ atoms on graphene. ${ }^{62}$ This theoretical result is also in good agreement with the experimental observation.

\section{Nitric oxide}

The investigation of the XUV light induced desorption process of nitric oxide from graphite is driven by the desire to learn more about the fundamental microscopic desorption dynamics of small molecules. NO can be detected with very high sensitivity and with internal state selection by resonantly enhanced $(1+$ 1) REMPI. It is the population distributions over the internal states of a product molecule which allow a deeper understanding of the microscopic dynamics. Figure 10 shows a rotational state resolved REMPI spectrum of the $\mathrm{A}^{2} \mathrm{~S}^{+}>\mathrm{X}^{2} \mathrm{P}$ $\left(\mathrm{v}^{00} 1 / 40,1\right) \mathrm{g}$-bands around $1 \frac{1 / 4}{226} \mathrm{~nm}$ arer excitation of the nitric oxide covered graphite surface by XUV light at $\mathrm{hn} 1 / 457.1 \mathrm{eV}$. It is evident that besides molecules in the vibrational ground state, also vibrationally excited molecules are detected. An analysis of the rotational state distribution yields a non-Boltzmann distribution with an average rotational energy in $\mathrm{v}^{00} 1 / 40$ of $\left\langle\mathrm{E}_{\mathrm{rot}}\right\rangle \frac{1 / 4}{4} 31 \mathrm{~cm}^{-1}$ or a rotational temperature of about $\left\langle\mathrm{E}_{\mathrm{rot}}\right\rangle / \mathrm{k} 1 / 4448 \mathrm{~K} .{ }^{63}$ For the vibrationally excited state the same distribution of the population in the rotational states is observed. The high intensity of the lines belonging to the $\mathrm{g}(1-1)$ band suggest already a considerable vibrational excitation. For these two vibrational states a vibrational 


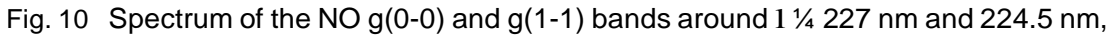
respectively, after desorption from $(\mathrm{NO})_{2} /$ graphite by XUV pulses of $\mathrm{hn} 1 / 457.1 \mathrm{eV}$.

temperature of about $\mathrm{T}_{\text {vib }} \mathrm{rv} 1580 \mathrm{~K}$ is found. Higher vibrational states could not be measured due to the limited beam time at the FEL facility. The translational energy of neutral NO molecules was obtained by changing the temporal delay between the FEL pulse (at hn $1 / 438 \mathrm{eV}$ ) and the detection laser pulse. A distribution is observed which can be 院tted by the combination of two Maxwellian velocity distributions. They show the temperature parameters of $\mathrm{T}_{\text {kin }} 1 / 4480 \mathrm{~K}$ and $\mathrm{T}_{\text {kin }} 1 / 41717 \mathrm{~K}$, and relative yields of $35 \%$ and $65 \%$, respectively. On average, a kinetic energy of $E_{\text {kin }} 1 / 4170 \mathrm{meV}$ is released in the desorption of neutral NO molecules. At this photon energy the desorption cross section was determined to about s $1 / 4(1.1 \pm 0.4) \$ 10^{-17} \mathrm{~cm}^{2}$. ${ }^{63}$ The desorption yield of NO from this $(\mathrm{NO})_{2} /$ graphite system exhibits a slightly nonlinear dependence on the XUV pulse intensity. ${ }^{63}$ Exponents between n $1 / 41.4$ and 3 have been observed in diff erent runs. Such a dependence would then enable the application of two-pulse correlated excitation of the system in order to learn more about the temporal behaviour of the excited system.

At surface temperatures below $\mathrm{T}_{\mathrm{S}} \mathrm{rv} 100 \mathrm{~K}$ nitric oxide condenses as cis-dimer $(\mathrm{NO})_{2}$ on graphite. ${ }^{64}$ The release of NO molecules thus has to be preceded by the dissociation of the NO dimer. Direct dissociative ionisation cross sections are about one order of magnitude lower than the desorption cross section measured in this study. ${ }^{65}$

Therefore, a direct photoexcitation of the adsorbate is unlikely, and an indirect excitation mechanism is suggested. The XUV photons are predominantly absorbed by the graphite substrate, thereby creating a cloud of hot electrons which interacts with the adsorbate. This interaction leads to a temporarily excited $(\mathrm{NO})_{2}{ }^{-}$dimer anion, where one NO molecule lies 蜜at on the surface. For the dimer anion the trans geometry is, however, energetically more favourable than the cis geometry. ${ }^{6}$ Therefore, the temporary formation of a dimer anion leads to both a strong rotational excitation of the leaving NO fragment due to this transition from a cis to a trans conformation of the dimer while in the excited state, as well as to a high vibrational excitation due to the transient negative ion, as in the case of metal substrates. Both conclusions are supported by the experimental observations. 


\section{Conclusion}

Femtosecond pulsed XUV radiation from free-electron laser sources is a valuable tool to induce reactive processes in molecular ices and elucidate the dynamics of such systems. Preliminary results of such reactions in water ice leading to cationic products have been presented. The high photon energy induces the formation of new species, in this case $\mathrm{D}_{2} \mathrm{O}_{2}$, which may act as a reservoir for further reactions. The observation of extremely slow $\mathrm{H}$ atoms from the chemisorbed state on graphite is really surprising and may have an in蜜uence on the understanding of the dynamics in accretion discs. The extension to other systems, such as doped or molecular ices where the neutral constituents themselves can be detected with internal state selection, will shed light onto the microscopic desorption dynamics. The study of the $(\mathrm{NO})_{2}$ thin 院 $1 m$ presented provides a 院rst glimpse of the wealth of information which can be obtained.

However, many interesting questions remain at present unanswered, including the velocity distribution of most of the oxygen containing cation products observed, and the relative roles of direct photoprocesses and processes induced by fast electrons liberated by the ionization of ice constituents. Also, the detection of neutral desorbing products, e.g. D atoms or OD radicals would contribute to a deeper understanding. Future experiments on VUV and XUV FEL facilities will allow us to address these questions and to contribute to a deeper microscopic understanding of reaction and desorption processes in complex ices.

\section{Acknowledgements}

The authors thank the FLASH team at HASYLAB for providing the XUV pulses, D. Gerlich, T. Olsen and J. Thrower for helpful discussions, and S. Eppenhoff and F. Wahlert for extensive technical support. The Bundesministerium für Bildung und Forschung provided partial 院nancial support via grant nos. 05 KS4PMC/8 and

05 KS7PM1 within the research program FSP 301 "FLASH" which is gratefully acknowledged. The European Union provided partial support via the FP7PEOPLE-ITN-2008 programme "LASSIE" no. 238258.

\section{References}

1 A. G. G. M. Tielens, Rev. Mod. Phys., 2013, 85, 1021.

2 E. Herbst and E. F. van Dishoeck, Annu. Rev. Astron. Astrophys., 2009, 47, 427.

3 R. J. Gould and E. E. Salpeter, Astrophys. J., 1963, 138, 393.

4 D. A. Williams and E. Herbst, Surf. Sci., 2002, 500, 823.

5 L. Hornekaer, A. Baurichter, V. Petrunin, D. Field and A. C. Luntz, Science, 2003, 302, 1943.

6 S. C. Creighan, J. S. A. Perry and S. D. Price, J. Chem. Phys., 2006, 124, 114701.

7 J. L. Lemaire, G. Vidali, S. Baouche, M. Chehrouri, H. Chaabouni and H. Mokrane, Astrophys. J., 2010, 725, L156.

8 G. Vidali, J. Low Temp. Phys., 2013, 170, 1.

9 J. S. Mathis, P. G. Mezger and N. Panagia, Astron. Astrophys., 1983, 128, 212.

10 S. S. Prasad and S. P. Tarafdar, Astrophys. J., 1983, 267, 603.

11 J. Najita, E. A. Bergin and J. N. Ullom, Astrophys. J., 2001, 561, 880.

12 T. Henning and D. Semenow, Chem. Rev., 2013, 113, 9016. 
13 J. S. Mathis, W. Rumpl and K. H. Nordsiek, Astrophys. J., 1977, 217, 425.

14 B. T. Draine and H. M. Lee, Astrophys. J., 1984, 285, 89.

15 D. Hollenbach, M. J. Kaufman, E. A. Bergin and G. J. Melnick, Astrophys. J., 2009, 690, 1497.

16 M. S. Westley, R. A. Baragiola, R. E. Johnson and G. A. Baratta, Nature, 1995, 373, 405.

17 S. R. Baggott, K. W. Kolasinski, L. M. A. Perdigao, D. Riedel, Q. Guo and R. E. Palmer, J. Chem. Phys., 2002, 117, 6667.

18 J. D. Thrower, D. J. Burke, M. P. Collings, A. Dawes, P. D. Holton, F. Jamme, P. Kendall, W. A. Brown, I. P. Clark, H. J. Fraser, M. R. S. McCoustra, N. J. Mason and A. W. Parker, Astrophys. J., 2008, 673, 1233.

19 E. C. Fayolle, M. Bertin, C. Romanzin, X. Michaut, K. I. Öberg, H. Linnartz and J.-H. Fillion, Astrophys. J., 2011, 739, L36.

20 K. I. Öberg, E. F. van Dishoeck and H. Linnartz, Astron. Astrophys., 2009, 496, 281.

21 K. I. Öberg, E. F. van Dishoeck, H. Linnartz and S. Andersson, Astrophys. J., $2010,718,832$.

22 D. McGonagle, L. M. Ziurys, W. M. Irvine and Y. C. Minh, Astrophys. J., 1990, 359, 121.

23 S. B. Charnley, S. D. Rogers and P. Ehrenfreund, Astron. Astrophys., 2001, 378, 1024.

24 D. T. Halfen, A. J. Apponi and L. M. Ziurys, Astrophys. J., 2001, 561, 244.

25 E. Congiu, H. Chaabouni, C. Laff on, P. Parent, S. Baouche and F. Dulieu, J. Chem. Phys., 2012, 137, 054713.

26 J. Bouwman, H. M. Cuppen, M. Steglich, L. J. Allamandola and H. Linnartz, Astron. Astrophys., 2011, 529, A46.

27 S. H. Cuylle, H. Linnartz and J. D. Thrower, Chem. Phys. Lett., 2012, 550, 79.

28 T. M. Orlando, A. B. Alexandrov and J. Herring, J. Phys. Chem. B, 2003, 107, 9370.

29 J. D. Thrower, M. P. Collings, F. J. M. Rutten and M. R. S. McCoustra, Chem. Phys. Lett., 2011, 505, 106.

30 A. G. M. Abdulgalil, D. Marchione, J. D. Thrower, M. P. Collings, M. R. S. McCoustra, F. Islam, M. E. Palumbo, E. Congiu and F. Dulieu, Philos. Trans. R. Soc. London, Ser. A, 2013, 371, 20110586.

31 K. Tiedtke, et al., New J. Phys., 2009, 11, 023029.

32 R. Mitzner, A. A. Sorokin, B. Siemer, S. Roling, M. Rutkowski, H. Zacharias, M. Neeb, T. Noll, F. Siewert, W. Eberhardt, M. Richter, P. Juranic, K. Tiedtke and J. Feldhaus, Phys. Rev. A: At., Mol., Opt. Phys., 2009, 80, 025401.

33 R. Mitzner, B. Siemer, M. Neeb, T. Noll, F. Siewert, S. Roling, M. Rutkowski, A. A. Sorokin, M. Richter, P. Juranic, K. Tiedtke, J. Feldhaus, W. Eberhardt and H. Zacharias, Opt. Express, 2008, 16, 19909.

34 M. Wöstmann, R. Mitzner, T. Noll, S. Roling, B. Siemer, F. Siewert, S. Eppenhoff , F. Wahlert and H. Zacharias, J. Phys. B: At., Mol. Opt. Phys., 2013, 46, 164005.

35 B. L. Henke, E. M. Gullikson and E. M. Davis, At. Data Nucl. Data Tables, 1993, $54,18$.

36 J. H. Hubell, W. J. Feigele, E. A. Briggs, R. T. Brown, D. T. Cromer and R. J. Howerton, J. Phys. Chem. Ref. Data, 1977, 4, 471.

37 L. Jeloica and V. Sidis, Chem. Phys. Lett., 1999, 300, 157. 
38 X. Sha and B. Jackson, Surf. Sci., 2002, 496, 318.

39 V. Hermann, B. D. Kay and A. W. Castleman Jr., Chem. Phys., 1982, 72, 185; F. Dong, S. Heinbuch, J. J. Rocca and E. R. Bernstein, J. Chem. Phys., 2006, 124, 224319.

40 J. E. Reutt, L. S. Wang, Y. T. Lee and D. A. Shirley, J. Chem. Phys., 1986, 85, 6928.

41 J. H. D. Eland, Adv. Mass Spectrom., 1974, 6, 917.

42 F. S. Ashmore and A. R. Burgess, J. Chem. Soc., Faraday Trans. 2, 1997, 73, 1247; S. N. Foner and R. L. Hudson, J. Chem. Phys., 1962, 36, 2676.

43 R. Atkinson, D. L. Baulch, R. A. Cox, J. N. Crowley, R. F. Hampson, R. G. Hynes, M. E. Jenkin, M. J. Rossi and J. Troe, Atmos. Chem. Phys., 2004, 4, 1461.

44 L. J. Stief and V. J. De Carlo, J. Chem. Phys., 1969, 50, 3.

45 R. G. Tonkyn, J. W. Winniczek and M. G. White, Chem. Phys. Lett., 1989, 164, 137.

46 NIST - JANAF Thermochemical Tables, $4^{\text {th }}$ edition, M. W. Chase, ed., 1998, Monograph 9, ISBN 1-56396-831-2.

47 R. T. Wiedmann, R. G. Tonkyn, M. G. White, K. Wang and V. McKoy, J. Chem. Phys., 1992, 97, 768.

48 R. L. Kelly, J. Phys. Chem. Ref. Data, 1987, 16 (suppl. 1), 1.

49 T. M. Orlando and M. T. Sieger, Surf. Sci., 2003, 528, 1.

50 X. N. Pan, A. D. Bass, J. P. Jay-Gerin and L. Sanche, Icarus, 2004, 172, 521.

51 B. Redlich, H. Zacharias, G. Meijer and G. von Helden, Surf. Sci., 2002, 502$503,325$.

52 B. Redlich, L. van der Meer, H. Zacharias, G. Meijer and G. von Helden, Nucl. Instrum. Methods Phys. Res., Sect. A, 2003, 507, 556.

53 B. Redlich, H. Zacharias, G. Meijer and G. von Helden, Phys. Chem. Chem. Phys., 2002, 4, 3448.

54 B. Redlich, H. Zacharias, G. Meijer and G. von Helden, J. Chem. Phys., 2006, 124, 044704.

55 B. Siemer, T. Hoger, M. Rutkowski, R. Treusch and H. Zacharias, J. Phys.: Condens. Matter, 2010, 22, 084013.

56 E. R. Latimer, F. Islam and S. D. Price, Chem. Phys. Lett., 2008, 455, 174.

57 L. Gavilan, J. L. Lemaire, G. Vidali, T. Sabri and C. Jäger, Astrophys. J., 2014, 781,79 .

58 B. Siemer, T. Olsen, T. Hoger, M. Rutkowski, C. Thewes, S. Düsterer, J. Schiøtz and H. Zacharias, Chem. Phys. Lett., 2010, 500, 291.

59 J. A. Misewich, T. F. Heinz and D. M. Newns, Phys. Rev. Lett., 1992, 68, 3737.

60 N. Rougeau, D. Teillet-Billy and V. Sidis, Chem. Phys. Lett., 2006, 431, 135.

61 L. Hornekaer, E. Rauls, W. Xu, Z. Sljivancanin, R. Otero, I. Stensgaard, E. Laegsgaard, B. Hammer and F. Besenbacher, Phys. Rev. Lett., 2006, 97, 186102 .

62 R. Frigge, T. Hoger, B. Siemer, H. Witte, M. Silies, H. Zacharias, T. Olsen and J. Schiøtz, Phys. Rev. Lett., 2010, 104, 256102.

63 B. Siemer, T. Hoger, M. Rutkowski, S. Düsterer and H. Zacharias, J. Phys. Chem. A, 2011, 115, 7356 .

64 J. P. Coulomb, J. Suzanne, M. Bienfait, M. Matecki, A. Thomy, B. Croset and C. Marti, J. Phys., 1980, 41, 1155.

65 J. A. R. Samson, T. Masuoka and P. N. Pareek, J. Chem. Phys., 1985, 83, 11.

66 B. Urban, A. Strobel and V. E. Bondybey, J. Chem. Phys., 1999, 111, 8939. 